\title{
Results of Stability Test in Subcooled Helium for the R\&D Coil of the LHD Helical Coil
}

\author{
S. Imagawa, N. Yanagi, Y. Hishinuma, T. Mito, K. Takahata, H. Chikaraishi, H. Tamura, A. Iwamoto, S. Hamaguchi, \\ K. Seo, S. Yamada, A. Nishimura, and O. Motojima
}

\begin{abstract}
Helical coils of the Large Helical Device are pool-cooled superconducting magnets. The operating current is restricted below about $90 \%$ of the design current because a normal-zone has propagated dynamically at several times at almost the same current. In order to estimate the effect of lowering temperatures on the cryogenic stability, an $R \& D$ coil was made of the same conductor. The cryogenic stability of the $R \& D$ coil was examined in saturated and subcooled helium. A normal-zone was initiated by a heater inserted between the conductor and the layer to layer spacer. The propagation was detected by voltage taps. In saturated helium of $4.4 \mathrm{~K}$ and $0.12 \mathrm{MPa}$, the minimum current to begin propagation is 10.7 to $10.8 \mathrm{kA}$. It becomes higher at the lower temperature, and it exceeds $11.7 \mathrm{kA}$ in subcooled helium of 3.5 $\mathrm{K}$ as a temperature inside the $R \& D$ coil.
\end{abstract}

Index Terms-Aluminum stabilizer, dynamic stability, minimum propagating current, subcooled helium.

\section{INTRODUCTION}

$\mathbf{H}$ ELICAL coils of the Large Helical Device (LHD) are pool-cooled superconducting magnets. The operating current is restricted below about $90 \%$ of the design current because normal-zones have infrequently propagated at almost the same current [1]. The normal-zones recovered after expanding to about $2 \mathrm{~m}$, except for a wide propagation at 11.45 $\mathrm{kA}$, which could not recover finally by deterioration of the cooling condition. A conductor of the coils consists of $\mathrm{NbTi} / \mathrm{Cu}$ strands, a pure aluminum stabilizer, and a copper housing. A normal-zone can propagate dynamically below the cold-end recovery current by additional heat generation due to the slow current diffusion into the thick stabilizer [2]. By a novel detection system of propagation with pick-up coils along the helical coils, it was revealed that the recovered normal-zones propagated to only one side except for the wide propagation. Concerning the last eight normal-zones, all were initiated at the bottom position of the coil and propagated to only the downstream side of the current. The propagation was stopped at the upper position, where the field is lower than the bottom.

Liquid helium is supplied from ten bottoms of the two helical coils, and the bubbles are exhausted to a header tank. The inlet temperature can be lowered to improve the cryogenic stability by installing an evacuated heat exchanger before the coils. In order to estimate the effect of lowering temperatures on the cryogenic stability, an R\&D coil was made of the same conductor as the helical coil. The cryogenic stability of the R\&D

Manuscript received October 20, 2003.

The authors are with the National Institute for Fusion Science, 322-6 Oroshi-cho, Toki, Gifu 509-5292, Japan (e-mail: imagawa@LHD.nifs.ac.jp).

Digital Object Identifier 10.1109/TASC.2004.830675
TABLE I

SPECIFICATIONS OF THE R\&D COIL

\begin{tabular}{ll}
\hline \hline Item & Design values \\
\hline Maximum field in coil & $6.9 \mathrm{~T}$ at $13.0 \mathrm{kA}$ \\
Winding number & 24 turns $\times 12$ layers \\
Inner radius of the innermost layer & $200 \mathrm{~mm}$ \\
Conductor size & $18.0 \mathrm{~mm} \times 12.5 \mathrm{~mm}$ \\
Gap between turns / Layers & $2.0 / 3.5 \mathrm{~mm}$ \\
Conductor length & $550 \mathrm{~m}$ \\
Inductance & $29 \mathrm{mH}$ \\
Weight of conductor / case & $0.86 / 1.7$ ton \\
\hline \hline
\end{tabular}

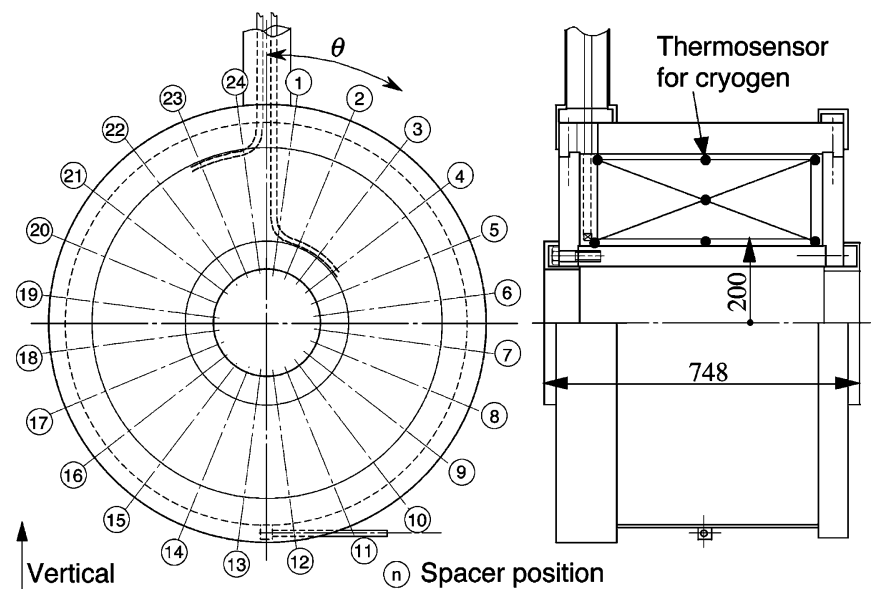

Fig. 1. Schematic drawing of the R\&D coil.

coil was examined in saturated and subcooled helium in December, 2002 and October, 2003. This paper intends to summarize the method and results of the cryogenic stability test.

\section{R\&D COIL AND EXPERIMENTAL METHODS}

Specifications and the schematic drawing of the $R \& D$ coil are shown in Table I and Fig. 1. The conductor was wound on a thick coil case by layer winding of 24 turns and 12 layers. An outer ring was divided into three parts, which were fixed by bolts to press the winding. After that, jointed parts were welded for leak-tightness. Electrically insulating spacers, thickness and width of which are 3.5 and $17 \mathrm{~mm}$, are settled on the inner ring by the pitch of $54 \mathrm{~mm}$. Turn to turn spacers are hooked on layer to layer spacers, as shown in Fig. 2. The wetting surface fraction of the first layer is $67 \%$, the same as the turns at the highest field of the LHD helical coil. The magnetic field becomes the highest at the middle turn of the first layer, which is the testing region for the cryogenic stability. The value is $6.9 \mathrm{~T}$ at $13 \mathrm{kA}$, the same 


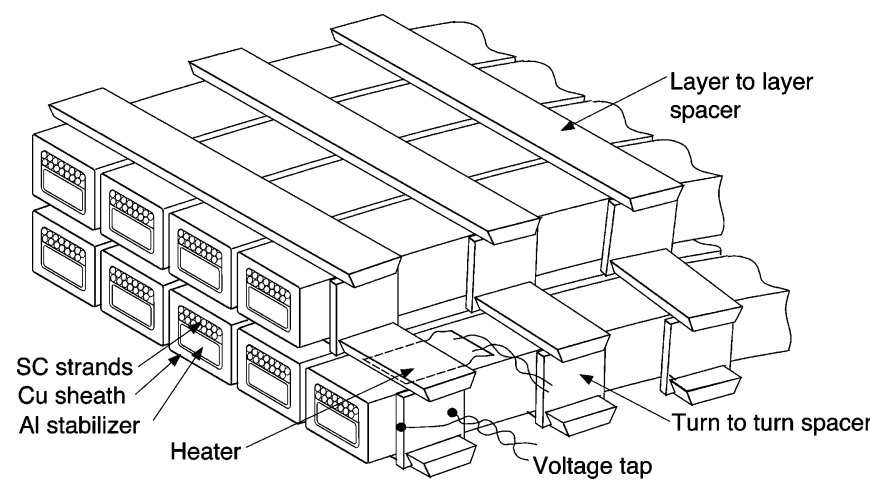

Fig. 2. Schematic drawing of the winding and sensors.

TABLE II

List OF VOLTAGE TAPS, THERMOMETERS, AND HEATER

\begin{tabular}{ll}
\hline Sensors et al. & Position (Tag Name) \\
\hline Balance Voltage & L7T24S1 and terminals (VB) \\
Voltage taps & L1T12S 1, 4, 7, 10, 13, 16, 19,22 (V1-8) \\
& L1T13S1, 4, 7, 10,12,13,14, 16, 19, 22 (V9-18) \\
& L1T14S1 (V19) \\
& L5T2S7, 10, 13, 16, 19 \\
\hline Voltage between turns & T8S24 and T10S24 (VL1) \\
& T10S24 and T12S24 (VL2) \\
& T13S24 and T15S24 (VL3) \\
& T15S24 and T17S24 (VL4) \\
\hline Thermometers & Near L1T1, $\theta=0,90,180,270$ deg. (TH1-4) \\
in cryogen & Near L1T13, $\theta=0,90,180,270$ deg. (TH5-8) \\
$(\theta:$ angle from the top) & Near L1T24, $\theta=0,90,180,270$ deg. (TH9-12) \\
& Center, $\theta=0,90,180,270$ deg. (TH13-16) \\
& Near L12T1, $\theta=0,90,180,270$ deg. (TH17-20) \\
& Near L12T13, $\theta=0,90,180,270$ deg. (TH21-24) \\
& Near L12T24, $\theta=0,90,180,270$ deg. (TH25-28) \\
L1T13S1, 7 (TC1, 2) \\
\hline Heaters on conductor & L1T12: S13(H1), 19 \\
& L1T13: S1, 7, 12, 13 \\
\hline & L5T2S13 \\
\hline \hline
\end{tabular}

(Note) Arabic numerals after "L", "T", and "S" mean the numbers of the layer, the turn, and the spacer position, respectively.

as the LHD helical coil. The length of the first layer is $1.3 \mathrm{~m}$ per turn.

The inlet pipe for cryogen is located at the bottom of the R\&D coil case. Although the diameter of the pipe is $10 \mathrm{~mm}$, a wide aperture of $20 \mathrm{~mm} \times 250 \mathrm{~mm}$ is drilled between the 12th and 13th spacer positions in the outer ring. The outlet pipe of the diameter of $100 \mathrm{~mm}$ is located at the top edge, in which a pair of coil-leads passes through.

Voltage taps are attached by solder on the side plane of the conductor at the testing region to detect the propagation of a normal-zone. The distance is $23 \mathrm{~mm}$. Since the half voltage of the coil in charging is induced near the end of the 7th layer, a voltage tap is attached on the 24th turn in the 7th layer. This middle voltage tap is utilized for the balance voltage. Tape heaters are inserted between the outer plane of the conductor and the layer to layer spacer to initiate a normal-zone. Thermo-sensors are installed not only on the conductors but also in the cryogen to examine the temperature distribution of subcooled helium in the coil. The list is shown in Table II.

The cryogenic stability of the R\&D coil was examined in saturated and subcooled helium. The flow diagram of the test facility [3] is shown in Fig. 3. Liquid helium is supplied from the

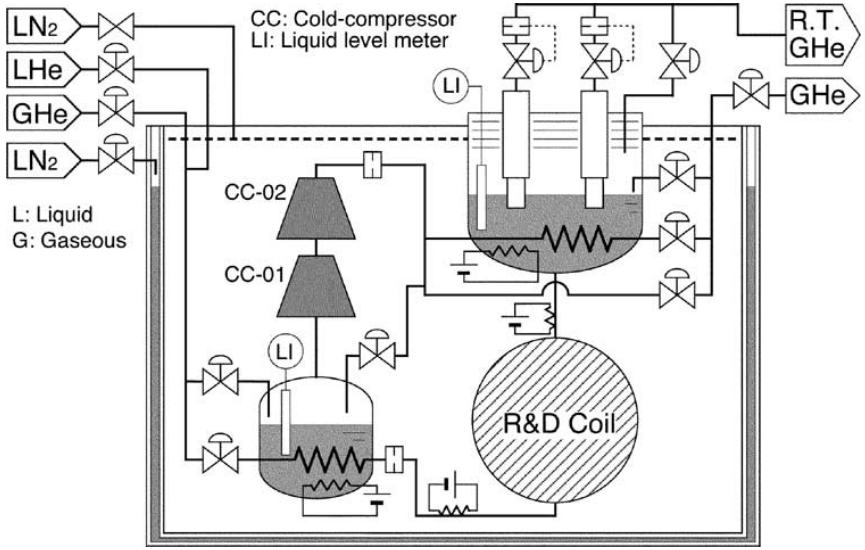

Fig. 3. Flow diagram of the test facility for the stability in subcooled helium.

bottom of the coil and exhausted through the coil-leads pipe to the header tank. The inlet helium is subcooled by a pre-cooler, which is evacuated by two cold-compressors. The pre-cooler is designed to be cooled down to $3.0 \mathrm{~K}$, that corresponds to the pressure of $0.024 \mathrm{MPa}$. In the first cool-down the R\&D coil was not covered with any thermal insulators to simulate the LHD helical coil. However, the estimated heat load to the coil was about $20 \mathrm{~W}$, that is much larger than the expected value. The lowest temperature inside the coil was $3.8 \mathrm{~K}$ at the highest flow rate of $9 \mathrm{~g} / \mathrm{s}$. The reason is considered to be the delay of cool-down of the inner vessel of the cryostat. Then, the $R \& D$ coil was covered by a thermal shield of copper plate with multi-layer insulators in the second cool-down. Thermal anchors of the shield are contacted to the coil-lead pipe. The heat load was reduced less than $7 \mathrm{~W}$, and the temperature inside the coil was lowered to $3.5 \mathrm{~K}$.

Normal-zones were initiated by the tape heater inserted between the conductor and the spacer. The heating duration is set to $20 \mathrm{~ms}$ to put as much energy as possible adiabatically. The heater at the bottom of the middle turn of the first layer was mainly used. Firstly, the minimum current for a normal-zone propagating more than the next voltage tap was surveyed with the maximum heating power of $100 \mathrm{~W}$. After that, the minimum heater power was surveyed by 100 A step.

\section{EXPERIMENTAL RESULTS}

Typical temperatures in the R\&D coil during the stability tests are shown in Fig. 4. The temperatures of the CERNOX thermosensors were calibrated in saturated helium at $0.12 \mathrm{MPa}$, and other temperatures were estimated by a fitting curve derived from the data of 20 calibrated sensors. The converting errors are estimated $0.015 \mathrm{~K}$ from 3.0 to $4.5 \mathrm{~K}$. Temperatures at TH19, $\mathrm{TH} 23$, and TH27 are lower than others because these are near the inlet for cryogen. TH17 located near the outlet was unfortunately broken. TH21 and TH25, that are located at higher position than the winding, indicate higher values than the others. Except for these special positions, the temperature difference inside the R\&D coil is very small [4]. The average temperatures are used as the coil temperatures.

In saturated helium at $0.12 \mathrm{MPa}$, the minimum currents to begin propagation are 10.6 to $10.8 \mathrm{kA}$, as shown in Fig. 5 . The currents become higher at the lower temperatures in subcooled helium. It is increased up to $11.7 \mathrm{kA}$ at $3.5 \mathrm{~K}$ as the coil 


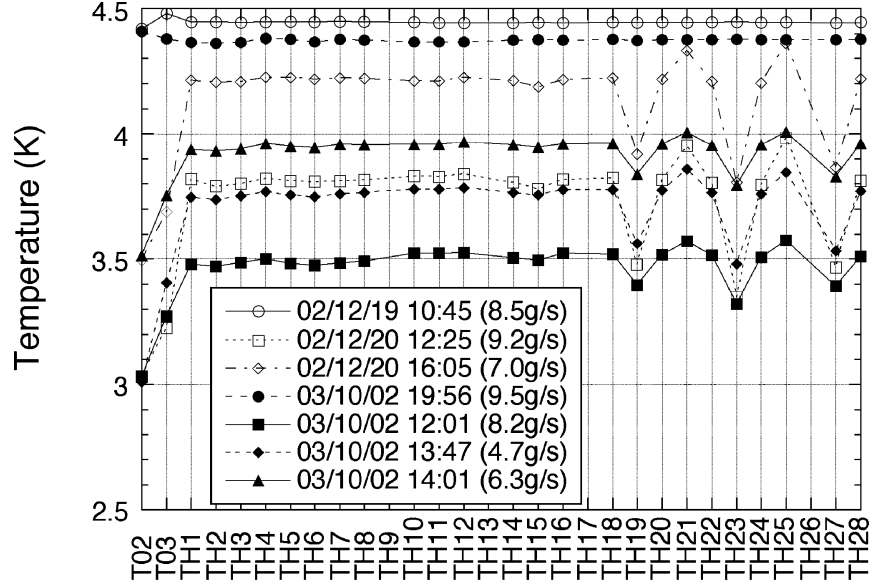

Position

Fig. 4. Temperature distribution in the $R \& D$ coil in saturated or subcooled helium. T02 and T03 are the temperatures at the outlet of the heat exchanger and the middle of the inlet pipe to the $R \& D$ coil.
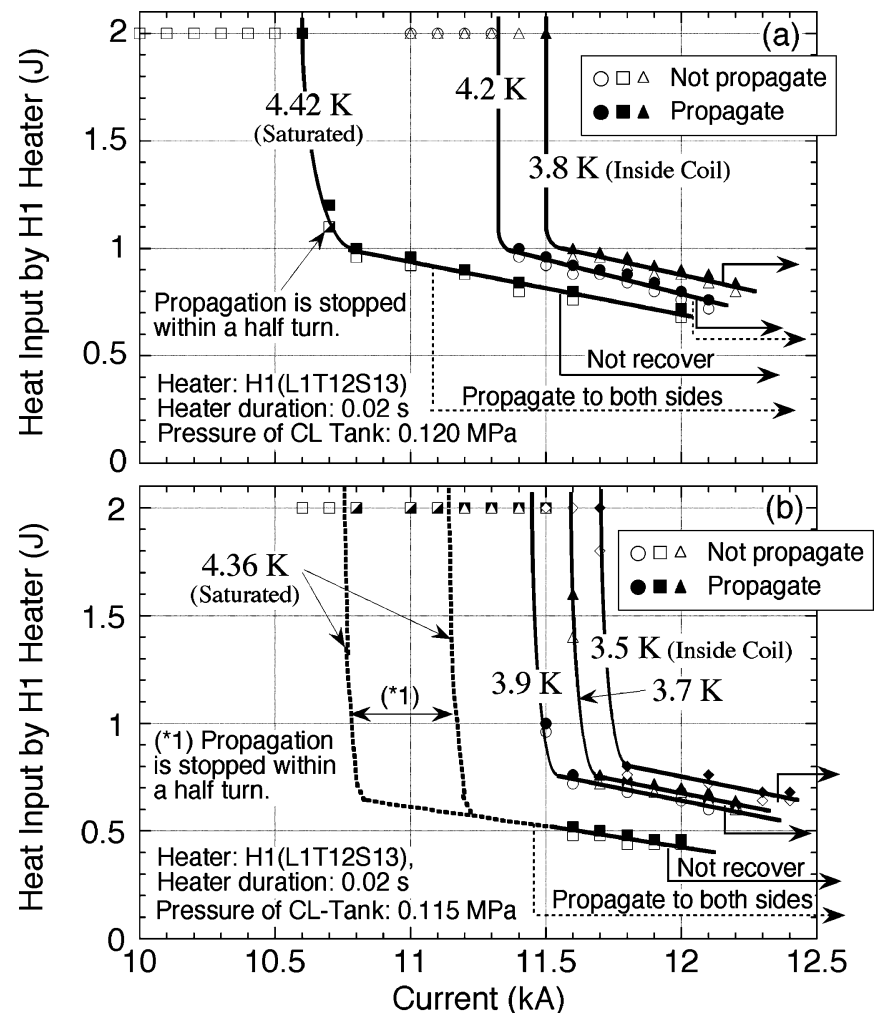

Fig. 5. Minimum heat input for the propagation of a normal-zone in the $R \& D$ coil in the first (a) and second cool-down (b).

temperature. At the currents a little higher than the threshold, normal-zones propagated to only the downstream side of the current. An example is shown in Fig. 6. A finite normal-zone propagated with recovery from the upstream side. The direction is same as the vector of $\boldsymbol{J} \boldsymbol{s} \times \boldsymbol{B}$, where the $\boldsymbol{J} \boldsymbol{s}$ is the transfer current from the superconducting strands to the aluminum stabilizer, and $B$ is the magnetic field [5].

At higher currents, normal-zones have propagated to both sides, as shown in Fig. 7. The upstream propagating velocity is almost half as downstream. In the cases of 11.1, 11.2, and $11.3 \mathrm{kA}$ in saturated helium at $0.12 \mathrm{MPa}$, the upstream propaga-

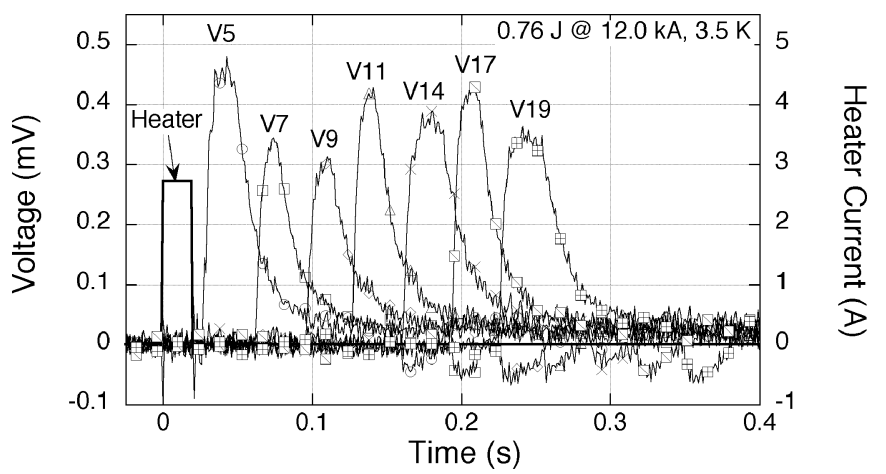

Fig. 6. Output of voltage taps during the one-side propagation of a normal-zone at $12.0 \mathrm{kA}$ in subcooled helium of $3.5 \mathrm{~K}$. The normal-zone propagated to only the downstream side of the current with recovery.
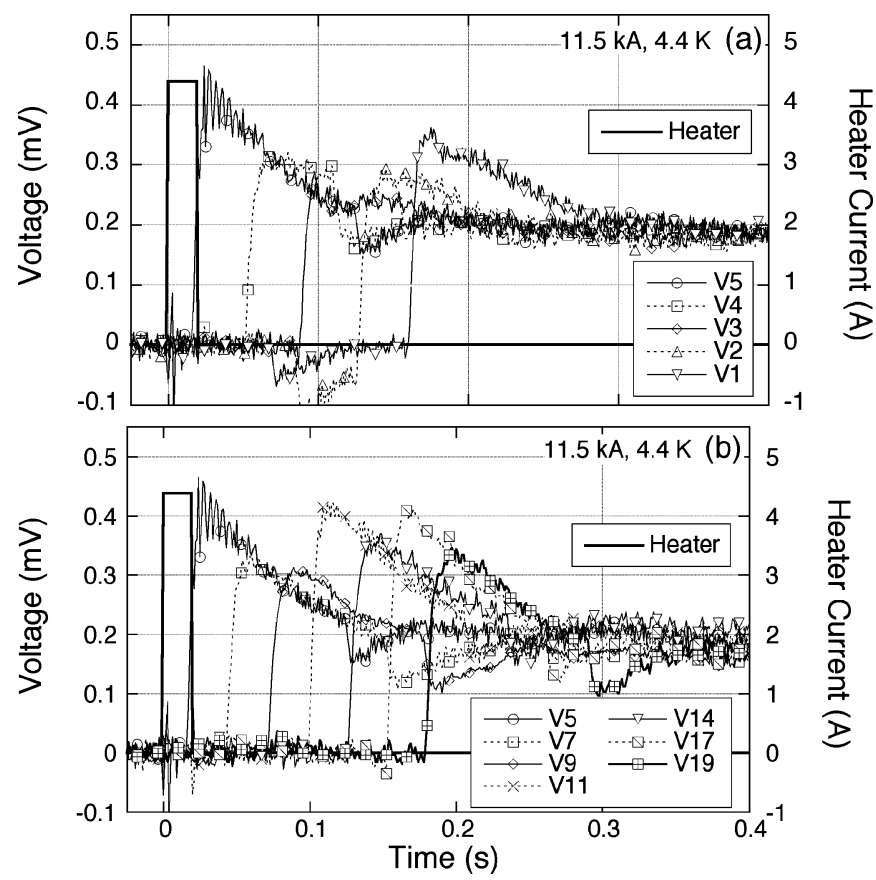

Fig. 7. Output of voltage taps during the both-side propagation of a normal-zone at $11.5 \mathrm{kA}$ in saturated helium at $0.120 \mathrm{MPa}$.

tion stopped within a half turn, and recovery started there. In the cases of 11.4 and $11.5 \mathrm{kA}$, even the upstream propagation continued to the next turn. The recovery started from the upper area in the middle turn. Namely, finite length of normal-zones propagated to both sides. At $11.6 \mathrm{kA}$, the normal-zones started recovering once from the middle turn, but the counter propagation was followed widely. The cooling condition was considered to be deteriorated by accumulation of helium bubbles. Fig. 8 shows an example of the voltages between turns in the case of recovering after the propagation to both sides in saturated helium. It shows that the recovery of the 15 th to 17 th turns was delayed. The recovering velocity may become slower and slower by accumulation of bubbles. In subcooled helium, the counter propagation was occurred at higher currents in spite of the one-side propagation.

Even the small difference in the temperatures of saturated helium in the second cool-down to the first one, the current for propagating over a half turn was increased up to $11.2 \mathrm{kA}$ from $10.7 \mathrm{kA}$, as shown in Fig. 5. The minimum current for propaga- 


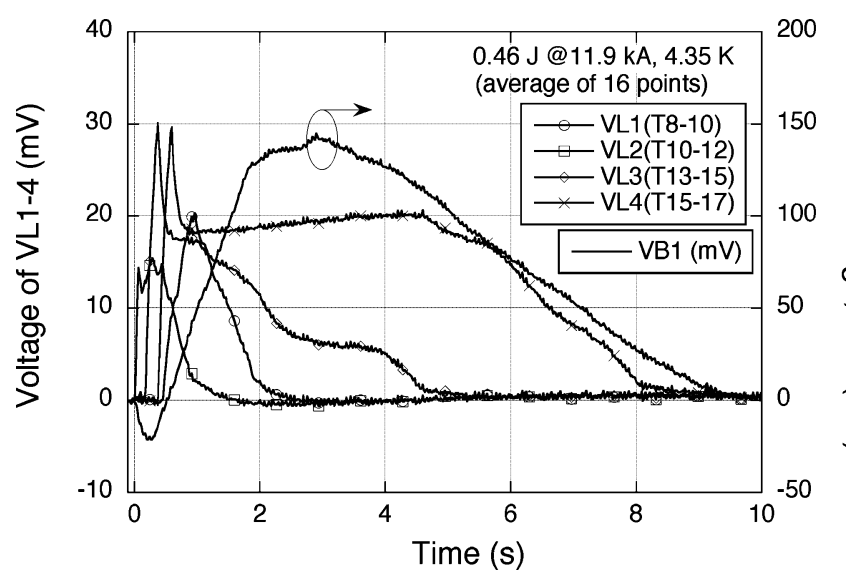

Fig. 8. Voltage between turns during the both-side propagation of a normal-zone and recovery from the middle turn in saturated helium at 0.115 MPa.

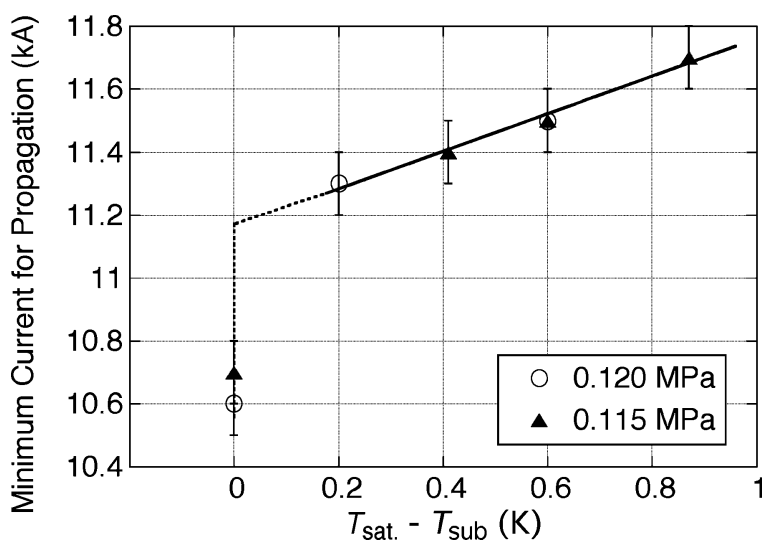

Fig. 9. Increase of the minimum currents for propagating with the degree of subcooling.

tion to both sides was also increased up to $11.5 \mathrm{kA}$ from $11.1 \mathrm{kA}$. The reason is not clear, but the amount of bubbles by steady heat load is possibly concerned. Besides, the minimum energy for propagation was different in the first and second cool-down. The thermal contact of heater may be improved by the cooling cycle.

The necessary heat input for initiating a normal-zone is enlarged by 10 to $20 \%$ in subcooled helium than in saturated helium. It becomes larger at the lower temperatures.

\section{DISCUSSION}

In subcooled helium the increment of the minimum currents to begin propagation is almost proportional to the degree of subcooling, as shown in Fig. 9. The current seems to increase discontinuously from the saturated to subcooled state. Quality of the saturated helium is possibly concerned. In other words, the quality of the first layer of the coil is apt to become worse by the accumulation of helium bubbles.

Fig. 10 shows these threshold currents compared with the currents of a normal-zone propagating in the LHD helical coil.

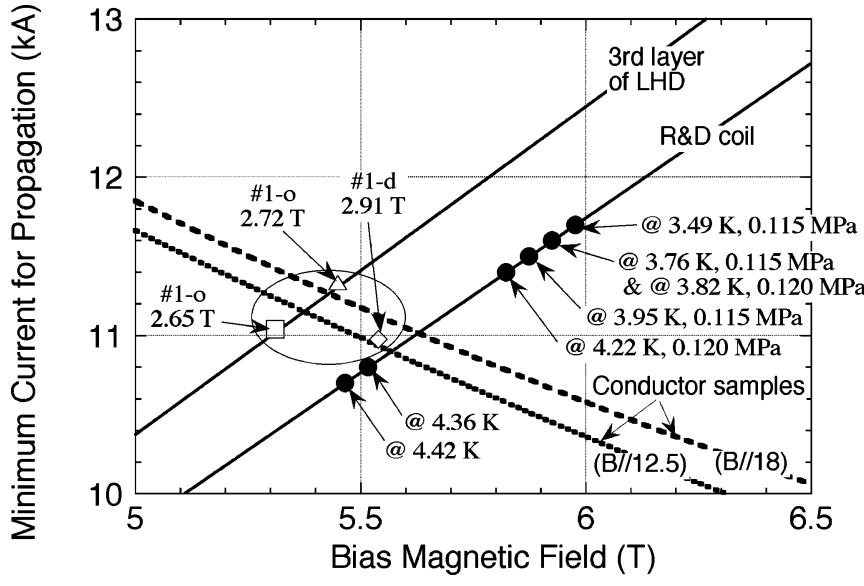

Fig. 10. Minimum current for propagation of a normal-zone in the composite conductor for the LHD helical coil.

Since the normal-zones are estimated to have been induced in the third layer in the LHD helical coil [1], the load line is shifted toward the lower field than the R\&D coil. If the LHD helical coils are cooled down to $3.5 \mathrm{~K}$, the operating current is expected to be increased to $12.0 \mathrm{kA}$.

\section{SUMMARY}

The cryogenic stability of the R\&D coil, the conductor of which is same as the LHD helical coil, was examined in saturated and subcooled helium. In saturated helium of 4.4 $\mathrm{K}$ and $0.12 \mathrm{MPa}$, the minimum current to begin propagation of a normal-zone is 10.7 to $10.8 \mathrm{kA}$. It becomes higher at the lower temperatures, and it exceeds $11.7 \mathrm{kA}$ in subcooled helium of $3.5 \mathrm{~K}$ as the coil temperature. The necessary heat input in subcooled helium is also increased by 10 to $20 \%$ than in saturated helium. The operating current of the LHD helical coils is expected to be increased up to $12.0 \mathrm{kA}$ by lowering the temperature of cryogen inside the coil to $3.5 \mathrm{~K}$.

\section{ACKNOWLEDGMENT}

The authors are indebted to the collaborators from Hitachi Co., Ltd. and Ishikawajima-Harima Heavy Industries Co., Ltd.

\section{REFERENCES}

[1] S. Imagawa, N. Yanagi, H. Sekiguchi, and T. Mito et al., "Consideration for the conductor motions in the helical coils of the Large Helical Device," IEEE Trans. Appl. Supercond., vol. 13, no. 2, pp. 1484-1487, June 2003.

[2] N. Yanagi, A. V. Gavrilin, and T. Mito et al., "Stability characteristics of the aluminum stabilized superconductor for the LHD helical coils," Advances in Superconductivity XI, pp. 991-994, 1999.

[3] Y. Hishinuma, S. Imagawa, and N. Yanaga et al., "Design and Operation of the Sub-Cooled R\&D System for the LHD Helical Coils," this conference.

[4] S. Hamaguchi and S. Imagawa, "Cooling Characteristics of R\&D Coil Cooled With Subcooled Helium for the LHD Helical Coils," this conference.

[5] N. Yanagi, S. Imagawa, and Y. Hishinuma et al., "Asymmetrical Normal-Zone Propagation Observed in the Aluminum-Stabilized Superconductor for the LHD Helical Coils," this conference. 